\title{
Wider die Stigmatisierung Suchtkranker
}

\section{Against the Stigmatization of Addicts}

Ein Patient wird am Montag um 12.00 Uhr von der Polizei einer körperlichen Durchsuchung unterzogen. Er hat eine Take-homeDosis von 6 Flaschen Methadon bei sich, kann aber keinen Substitutionsausweis vorlegen. Daraufhin entleert die Polizei ohne Rücksprache mit der Praxis alle Flaschen.

Ein Drogenabhängiger stellt sich mit Atemnot in der Notaufnahme eines Krankenhauses vor. Als sich herausstellt, dass er drogenabhängig ist, wird er ohne weitere Untersuchungen weggeschickt. Durch das Engagement der Mutter und den Kontakt zu einem suchtmedizinisch tätigen Kliniker wird der Patient schließlich aufgenommen. Er leidet an einer Lungenembolie. Er kommt für zwei Wochen auf die Intensivstation, ist z.T. beatmungspflichtig. Er überlebt.

Ein suchtmedizinisch tätiger Kollege wird regelhaft von seinen Kollegen gefragt, ob er es denn nötig habe, solche Patienten (gemeint sind abhängige Patienten) zu behandeln.

Das von der Norm Abweichende unterliegt immer der Gefahr, ausgegrenzt zu werden. Diese Ausgrenzung kann sich auf seelische und körperliche Erkrankung beziehen, kann Armut oder auch sexuelle Orientierung zum Inhalt haben. Die der Stigmatisierung unterworfenen Merkmale sind prinzipiell beliebig. Es gibt keine geschichtliche oder regionale Konstanz: Was vor hundert Jahren ein Stigma war, gilt heute als normal, was in der einen Gesellschaft als abstoßend gekennzeichnet ist, genießt in einer anderen Gesellschaft höchste Wertschätzung.
Die oben genannten Beispiele zeigen, dass sowohl suchtkranke Menschen als auch deren Behandler tagtäglich vielfältige Diskriminierungen erleben. Der diesjährige Kongress der Deutschen Gesellschaft für Suchtmedizin (DGS) widmete sich unter dem Titel „Suchtbekämpfung oder Bekämpfung der Süchtigen“ der Stigmatisierung.

Um die Bemühungen gegen die Stigmatisierung suchtkranker Menschen und deren Behandler über den Kongress hinaus dauerhaft fortzuführen, wurde im November in Berlin der Verein AST (AntiSTigma) gegründet. AST hat sich zur Aufgabe gemacht, mit Aktionen und Aufklärung aktiv gegen die gesellschaftliche Diskriminierung Suchtkranker vorzugehen. Vorurteile sollen abgebaut werden. Der Verein will eine positive Veränderung im Bewusstsein und Verhalten gegenüber diesen Betroffenen bewirken. Um eine Breitenwirkung zu erzielen, wurde ein Freundeskreis ins Leben gerufen. Diesem haben sich bisher u.a. der Musiker Konstantin Wecker, die Regisseure Dieter Wedel und Elmar Goerden, der Filmproduzent Jürgen Kriwitz, Fußballtrainer Ottmar Hitzfeld und über 100 Unterstützer aus den verschiedensten gesellschaftlichen Bereichen angeschlossen. Ein Anstecker in Form eines ASTes dient als Symbol und signalisiert Unterstützung im Kampf gegen die Diskriminierung.

Wir hoffen, dass auch die Arbeiten, die in dieser Ausgabe der Suchttherapie vorgestellt werden, ihren Teil zur Entstigmatisierung suchtkranker Menschen beitragen.

Dr. Jens Reimer, Hamburg

Dr. Greif Sander, Sehnde/Hannover 